\title{
Hypoxic-Ischemic Oligodendroglial Injury in Neonatal Rat Brain
}

\author{
YIQING LIU, FAYE S. SILVERSTEIN, ROBERT SKOFF, AND JOHN D.E. BARKS \\ Departments of Pediatrics [Y.L., F.S.S., J.D.E.B.] and Neurology [F.S.S.], University of Michigan, Ann \\ Arbor, Michigan, U.S.A.; and Department of Anatomy and Cell Biology, Wayne State University, Detroit, \\ Michigan 48201, U.S.A. [R.S.]
}

\begin{abstract}
Neonatal periventricular white matter injury is a major contributor to chronic neurologic dysfunction. In a neonatal rat stroke model, myelin basic protein (MBP) immunostaining reveals acute periventricular white matter injury. Yet, the extent to which myelin proteins can recover after neonatal hypoxicischemic injury is unknown. We developed a quantitative method to correlate the severity of the hypoxic-ischemic insult with the magnitude of loss of MBP immunostaining. Seven-day-old (P7) rats underwent right carotid ligation, followed by exposure to $8 \%$ oxygen for 1, 1.5, 2, or $2.5 \mathrm{~h}$. On both P12 and P21, white matter integrity was evaluated by densitometric analysis of MBP immunostaining, and the amount of tissue injury was evaluated by morphometric measurements of cerebral hemisphere areas. The most severe hypoxic-ischemic insults $(2.5 \mathrm{~h})$ elicited marked reductions in MBP immunostaining ipsilaterally on both P12 and $\mathrm{P} 21$. In contrast, in mildly lesioned animals (1.5 h), MBP immunostaining was reduced ipsilaterally on P12, but 2 wk after lesioning, on P21, there was a substantial restoration of MBP
\end{abstract}

\section{ABSTRACT}

immunostaining. The restoration in MBP immunostaining could reflect either functional recovery of injured oligodendroglia or proliferation and maturation of oligodendroglial precursors. Our data demonstrate that quantitative measurement of MBP immunostaining provides a sensitive indicator of acute oligodendroglial injury. Most importantly, we show that in this neonatal rodent stroke model, restoration of myelin proteins occurs after moderate, but not after more severe, cerebral hypoxia-ischemia.

(Pediatr Res 51: 25-33, 2002)

$\mathbf{P}$, postnatal day

\section{Abbreviations}

MBP, myelin basic protein

HI, hypoxia-ischemia

Ab, antibody

(R:L) $)_{\text {MBP }}$, ratio of right-to-left hemispheric MBP

(R:L) $)_{\text {AREA }}$, ratio of right-to-left hemispheric area
Considerable clinical and experimental data demonstrate that immature oligodendroglia are highly susceptible to $\mathrm{HI}$ injury (1-5). There is also growing recognition that oligodendroglial injury in the immature nervous system may have profound adverse effects on neuronal development (6). Among the mechanisms implicated as contributing to the vulnerability of immature oligodendroglia to $\mathrm{HI}$ are increased susceptibility to excitotoxicity $(1,7)$, oxidative stress $(2)$, and inflammation (8-9), and propensity for induction of apoptosis (10).

In a widely used neonatal rat stroke model, elicited by unilateral carotid artery ligation and timed exposure to moderate hypoxia ( $8 \%$ oxygen) in 7 -d-old (P7) rats, ipsilateral white matter injury occurs, together with more widespread tissue injury (11-15). In this model, lengthening the duration of

Received May 3, 2001; accepted September 5, 2001.

Correspondence and reprint requests: F.S. Silverstein, M.D., University of Michigan, Room 8301 MSRB3, Box 0646, 1150 W. Medical Center Drive, Ann Arbor, MI 48109-0646, U.S.A.; e-mail: fsilvers@med.umich.edu

Supported by US Public Health Service grants NS 35059 (F.S.S. and J.D.E.B.) and NS 37036 (J.D.E.B.), and a grant (RG2579) from the National Multiple Sclerosis Society (R.S.). hypoxic exposure results in a progressive increase in the severity of brain damage (12); the temporal threshold to elicit tissue injury is at approximately $1.5 \mathrm{~h}$, and more prolonged $\mathrm{HI}$ $(2-2.5$ h) commonly elicits extensive neuronal loss in the ipsilateral cortex, hippocampus, striatum, and thalamus (13). Additional neuropathologic features of the HI lesion include an acute and sustained microglial and monocyte infiltrate $(13,14)$, reactive gliosis $(13,15)$, and the evolution of cortical cavitary infarcts (13).

Although the original description of histopathology in this model documented the vulnerability of white matter to injury, subsequent work in this model has focused on the pathophysiology of neuronal damage and the development of therapeutic strategies for neuroprotection. Until recently, modulation of white matter injury has been relatively neglected experimentally; one practical issue that has limited such studies relates to the difficulties inherent in the quantification of oligodendroglial injury.

Two complementary approaches have been used to assess oligodendroglial integrity experimentally: evaluation of 
changes in numbers of oligodendroglial cell bodies, and evaluation of changes in myelin-specific mRNAs and proteins. Recent data suggest that measures of myelin-specific mRNAs and proteins yield informative indices of acute oligodendroglial injury. In neonatal mice, HI lesioning resulted in rapid reductions in expression of MBP mRNA and protein in the lesion territory (5). In P7 rats, unilateral carotid artery ligation, followed by relatively brief $(1 \mathrm{~h})$ exposure to $6 \%$ oxygen, resulted in relatively selective acute oligodendroglial injury, manifest by evidence of DNA damage and loss of MBP immunostaining in the ipsilateral external capsule (7), with sparing of adjacent cortex; the efficacy of protective interventions was evaluated by scoring intensity of MBP immunostaining in drug-treated and control animals.

Based on these findings, in this study we focused on analysis of MBP immunostaining as an indicator of acute oligodendroglial injury in neonatal rodent brain. We developed a computerized image-analysis-based semiquantitative method for measurement of MBP immunostaining in neonatal rodent brain. This method was applied to assess white matter injury in neonatal rodents that underwent $\mathrm{HI}$ lesioning. Our initial goal was to evaluate the relationship between the duration of the HI insult and the severity of acute oligodendroglial injury, based on evaluation of MBP immunostaining at $5 \mathrm{~d}$ after lesioning. In addition, to determine whether any observed reductions in MBP immunostaining were sustained, we also evaluated outcome after a 2 -wk recovery period.

\section{METHODS}

Animal lesioning. We used the methods originally described by Rice et al. (11), with minor modifications, as previously reported (16). Seven-day-old Sprague-Dawley rats were deeply anesthetized by methoxyflurane inhalation, and the right carotid artery was isolated and double-ligated. Animals were allowed to recover from anesthesia in incubators, maintained at ambient temperature $36^{\circ} \mathrm{C}$, for $1 \mathrm{~h}$; then they were placed in Plexiglas chambers, partially submerged in $36^{\circ} \mathrm{C}$ water baths, and exposed to $8 \% \mathrm{O}_{2}$ for $1-2.5 \mathrm{~h}$. They recovered in thermoregulated incubators for up to $60 \mathrm{~min}$, and were then returned to the dams. This procedure results in a marked ipsilateral decline in cerebral blood flow during hypoxic exposure, with a return to normal blood flow after the cessation of hypoxic exposure (17).

In preliminary experiments, we found that right carotid artery ligation, followed by $1.5 \mathrm{~h}$ exposure to $8 \% \mathrm{O}_{2}$, resulted in substantial loss of MBP immunostaining ipsilaterally $5 \mathrm{~d}$ after lesioning, although overall tissue integrity was relatively preserved; thus, we focused on this lesioning protocol in subsequent experiments. Two groups of animals were included in this study. Group 1, evaluated $5 \mathrm{~d}$ after lesioning on P12, included three unlesioned controls and 19 lesioned animals that all underwent carotid artery ligation on $\mathrm{P} 7$, followed by $1 \mathrm{~h}(n=3), 1.5 \mathrm{~h}(n=$ $8), 2 \mathrm{~h}(n=3)$, or $2.5 \mathrm{~h}(n=5)$ exposure to $8 \% \mathrm{O}_{2}$. Group 2, evaluated 2 wk after lesioning on P21, included two controls and 17 lesioned animals that all underwent carotid artery ligation on P7, followed by $1 \mathrm{~h}(n=3), 1.5 \mathrm{~h}(n=6), 2 \mathrm{~h}(n=4)$, or $2.5 \mathrm{~h}$ $(n=4)$ hypoxic exposure. Animals were killed with a lethal overdose of chloral hydrate $(450 \mathrm{mg} / \mathrm{kg})$, followed by perfusion fixation with $4 \%$ paraformaldehyde. Experimental protocols were approved by the University of Michigan Committee on Use and Care of Animals. All efforts were made to minimize animal suffering and the number of animals used.

Immunocytochemistry. Brains were removed, postfixed in $4 \%$ paraformaldehyde $\left(16 \mathrm{~h}\right.$, at $\left.4^{\circ} \mathrm{C}\right)$, and cryoprotected in graded $(5 \%$ to $25 \%)$ sucrose solutions in PBS, as previously described (14). Coronal sections (16 $\mu \mathrm{m}$, at $64-\mu \mathrm{m}$ intervals) were collected on gelatin-coated slides; at least six sections/ brain, three at the level of the mid-striatum and three at mid dorsal hippocampus, were assayed.

Sections were washed (PBS, $5 \mathrm{~min}$ ) and preincubated in $20 \%$ normal horse serum in PBS with $0.5 \%$ Triton X-100. Both $\mathrm{Ab}$ were diluted in PBS with $0.5 \%$ Triton $\mathrm{X}-100$. Sections were incubated with the primary $\mathrm{Ab}$ (anti-MBP, mouse monoclonal, SMI-94, Sternberger, Baltimore, MD, U.S.A.) 1:400, overnight at $4^{\circ} \mathrm{C}$. Sections were washed and incubated with a biotinylated secondary $\mathrm{Ab}$ (rat adsorbed horse anti-mouse, Vector Laboratories, Burlingame, CA, U.S.A.) 1:200, for $1 \mathrm{~h}$ at room temperature. Sections were washed again, and a Vectastain ABC Elite Kit was used to amplify signal (according to manufacturer's instructions, $30 \mathrm{~min}$, at room temperature), followed by chromogenic detection with stable diaminobenzidine (Research Genetics, Huntsville, AL, U.S.A.) for $5 \mathrm{~min}$ at room temperature. Sections were dehydrated in graded ethanols, and cover-slipped with Permount. Control samples in which an equal amount of mouse $\mathrm{IgG}$ was substituted for the primary $\mathrm{Ab}$ were included in each assay, and were uniformly negative.

Image analysis methods. A computerized video-camerabased image-analysis system (with NIH Image software, public domain, available at: http://rsb.info.nih.gov/nih-image/) was used for densitometry and cross-sectional area measurements. All available sections (at least five per brain) were analyzed; only sections with obvious technical artifacts related to the staining procedure were excluded. For densitometry, unaltered TIFF images were digitized, segmented (using a consistent arbitrary threshold $-50 \%$ ), and binarized (black versus white); then, total black pixels per hemisphere were counted, and average values were calculated per brain, expressed as pixels per hemisphere. Hemisphere areas were also outlined and measured in each section that was analyzed by densitometry. Photomontages were prepared from images processed using Photoshop 5.0 (Adobe, Mountain View, CA, U.S.A.).

Data analysis. Because there were interassay differences in the intensity of MBP immunostaining, no attempt was made to compare absolute OD values among experimental groups. OD values were expressed as ratios of right-to-left hemispheric measurements; for each brain sample, $(\mathrm{R}: \mathrm{L})_{\mathrm{MBP}}$ of pixels per right hemisphere to pixels per left hemisphere was calculated.

In this neonatal stroke model, because injury is incurred at a time of rapid brain growth, cerebral hemisphere cross-sectional area measurements, obtained as early as $5 \mathrm{~d}$ after lesioning, provide a sensitive indicator of the extent of tissue damage (16). In these experiments, to assess the severity of tissue injury, for each brain sample corresponding bihemispheric area ratios were calculated $\left[(\mathrm{R}: \mathrm{L})_{\mathrm{AREA}}=\right.$ mean right hemisphere area to mean left hemisphere area]. 

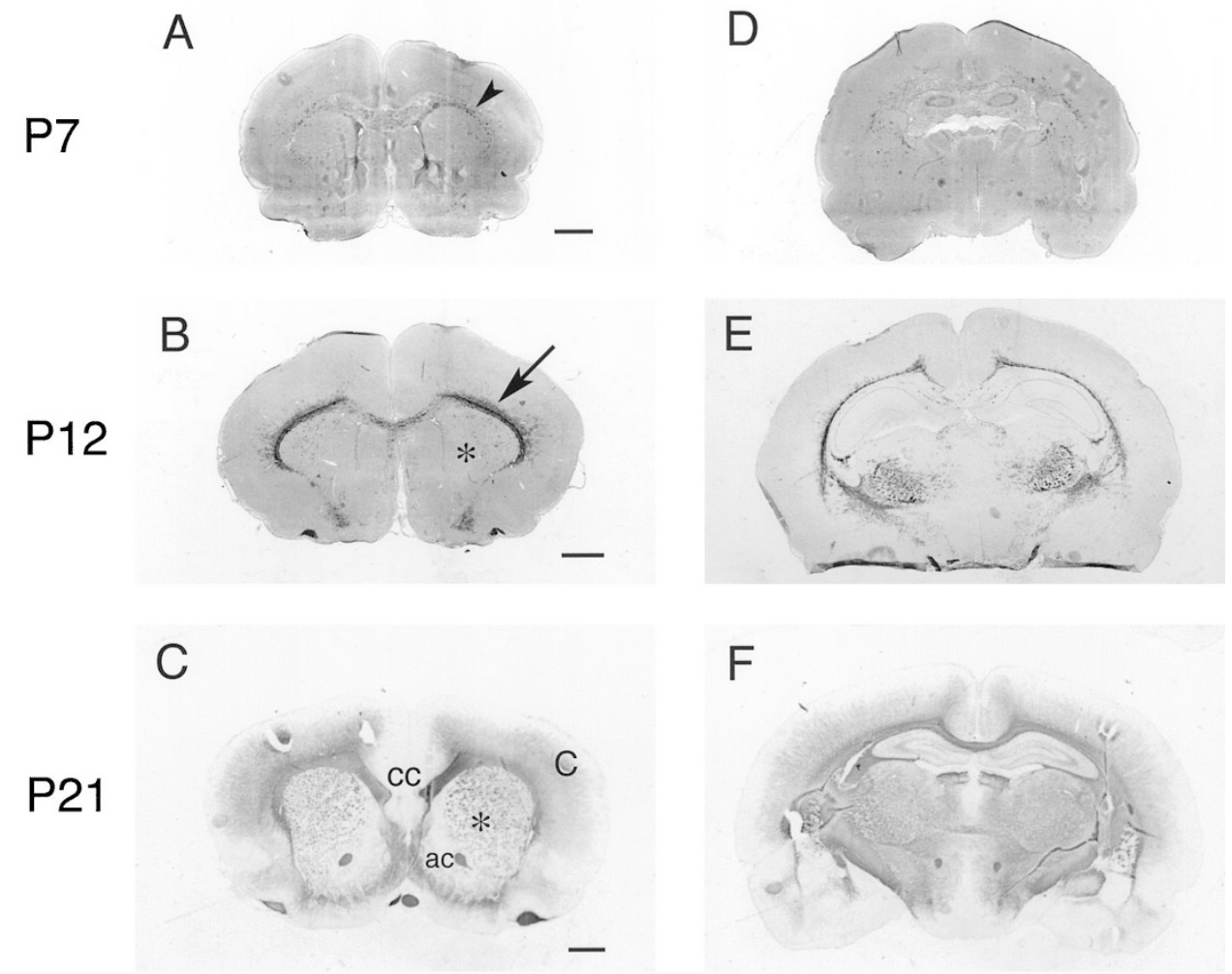

Figure 1. Maturational changes in MBP immunostaining in rat brain from P7 to P21. MBP immunocytochemistry assays were performed, as described in "Methods," in tissue samples from normal P7, P12, and P21 rat brain. At the level of the striatum on P7 $(A)$, MBP immunostaining is barely discernible in the external capsule (arrowhead); on P12 (B), MBP immunostaining is most intense in the external capsule (arrow), and subtle periaxonal staining can be discerned within the striatum $\left(^{*}\right)$; on P21 $(C)$, there is more pronounced MBP immunostaining in the corpus callosum ( $c c$ ), the anterior commissures $(a c)$, the striatum, and extending into the cortex. At the level of the hippocampus, similar maturational trends are evident from P7-21 $(D-F)$. Scale bars, $1 \mathrm{~mm}$.

Data were analyzed using a microcomputer-based statistics and graphing program (GraphPad, Prism 2.01, Mountain View, CA, U.S.A.). Data from the P12 and P21 outcome groups were first analyzed independently. In animals of each age, differences among lesioning protocols (i.e. duration of $\mathrm{HI}$ ) were compared, using a nonparametric ranking test (Kruskal-Wallis). Because preliminary histopathologic evaluation and graphic analysis of trends suggested that in mildly lesioned animals $(1.5 \mathrm{~h} \mathrm{HI})$, the outcomes differed markedly between P12 and P21, (R:L) MBP values were compared at these two time intervals, using a MannWhitney nonparametric ranking test. In addition, to assess the equivalence of overall lesion severity between groups, the corresponding (R:L) $)_{\text {AREA }}$ values were also compared using a MannWhitney nonparametric ranking test.

\section{RESULTS}

In the developing rat brain during the second and third postnatal weeks, there is a substantial increase in MBP immunostaining. Figure 1 illustrates the distribution of MBP immunostaining in normal P7, P12, and P21 rat brain, at the levels of striatum (Fig. 1, $A-C$ ), and mid-dorsal hippocampus (Fig. 1, $D-F)$. On P7, MBP immunostaining is barely detectable. On P12, MBP immunostaining is readily identified, concentrated in white matter tracts of the external and internal capsules; in the striatum periaxonal immunostaining can also be discerned. On P21, there is a further increase in MBP immunostaining, but the widespread adult distribution has not yet attained; note more intense immunostaining, both within white matter tracts and also extending into adjacent gray matter regions.

Table 1 presents regional cross-sectional area measurements, and calculated mean (R:L) $)_{\text {AREA }}$ values in all lesion groups. Comparison of cerebral hemisphere areas in the unlesioned P12 and P21 groups illustrates the substantial increase

Table 1. Cross-sectional area measurements: an indicator of ipsilateral cerebral hemisphere injury

\begin{tabular}{lcccc}
\hline & $n$ & $\begin{array}{c}\text { Left } \\
\text { hemisphere } \\
\left(\mathrm{mm}^{2}\right)\end{array}$ & $\begin{array}{c}\text { Right } \\
\text { hemisphere } \\
\left(\mathrm{mm}^{2}\right)\end{array}$ & $(\mathrm{R}: \mathrm{L})_{\mathrm{AREA}}{ }^{*}$ \\
\hline P12 outcome group & & & & \\
Normal & 3 & $28.3 \pm 0.1$ & $27.6 \pm 0.2$ & $0.98 \pm 0.0$ \\
$1.0 \mathrm{~h} \mathrm{HI}$ & 3 & $28.9 \pm 2.5$ & $28.5 \pm 1.4$ & $0.99 \pm 0.0$ \\
1.5 h HI & 8 & $31.4 \pm 1.3$ & $26.4 \pm 1.1$ & $0.84 \pm 0.3$ \\
2.0 h HI & 3 & $30.6 \pm 0.8$ & $26.6 \pm 0.6$ & $0.87 \pm 0.0$ \\
2.5 h HI & 5 & $30.7 \pm 1.1$ & $22.4 \pm 1.4$ & $0.73 \pm 0.1$ \\
P21 outcome group & & & & \\
Normal & 2 & $39.0 \pm 2$ & $37.3 \pm 2.5$ & $0.96 \pm 0.0$ \\
1.0 h HI & 3 & $41.2 \pm 0.6$ & $38.3 \pm 0.9$ & $0.93 \pm 0.0$ \\
1.5 h HI & 6 & $42.5 \pm 0.7$ & $38.3 \pm 1.4$ & $0.90 \pm 0.0$ \\
2.0 h HI & 4 & $41.4 \pm 1.0$ & $33.2 \pm 2.6$ & $0.80 \pm 0.1$ \\
2.5 h HI & 4 & $40.1 \pm 1.8$ & $21.7 \pm 4.9$ & $0.62 \pm 0.1$ \\
\hline
\end{tabular}

Cross-sectional areas of each cerebral hemisphere were measured in all tissue sections used for analysis of MBP immunostaining (see "Methods").

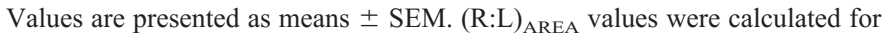
each brain sample and means are presented. P12 and P21 (R:L) $)_{\text {AREA }}$ values were analyzed independently; $* p<0.05$ in P12, $p<0.01$ in P21, comparing $(\mathrm{R}: \mathrm{L})_{\text {AREA }}$ values in each group with Kruskal-Wallis test. 
in brain size that occurs during this 10 -d period (mean hemisphere area increasing from 28 to $38 \mathrm{~mm}^{2}$ ); in the lesioned animals, left hemisphere area values did not differ from values in age-matched controls. These data confirm that in both the P12 and P21 outcome groups, the duration of HI determined the severity of tissue loss. There was negligible injury in the 1-h HI groups; there was substantial (>25\%) ipsilateral hemisphere damage in both 2.5 -h HI groups.

Figure 2 compares MBP immunostaining, at the level of the mid-striatum, in eight lesioned animals, evaluated on P12 (Fig. 2, $A, C, E, G$ ) or on P21 (Fig. 2, $B, D, F, H$ ). Each row compares animals that underwent the same duration of HI (1, $1.5,2$, or $2.5 \mathrm{~h}$ ). Overt infarction evolved only in the 2.5 -h HI animals. Comparison of MBP immunostaining in the P12 animals reveals a progressive ipsilateral loss of MBP immunostaining in the ipsilateral external capsule and striatum. In the P21 animals substantial asymmetry in MBP immunostaining is apparent only in the most severely lesioned animal.
Figure 3 illustrates corresponding MBP immunostained sections, at the level of dorsal hippocampus and thalamus; overall trends are similar. On P12, there is substantial and progressive loss of ipsilateral MBP immunostaining in the external and internal capsules of animals that underwent $1.5,2$, or $2.5 \mathrm{~h} \mathrm{HI}$; on P21, MBP immunostaining is relatively preserved, except in the most severely lesioned animal. Note that the distribution and intensity of MBP immunostaining in the left hemisphere of lesioned animals (Figs. 2 and 3) is similar to that of unlesioned age-matched controls (Fig. 1).

Figure 4 illustrates the features of MBP immunostaining, at higher magnification, in a representative P12 animal that underwent $1.5 \mathrm{~h}$ HI on P7. In Figure 4, $A$, note that there is subtle right striatal atrophy, although overall tissue integrity is preserved; yet there is widespread ipsilateral loss of MBP immunostaining (Fig. 4, $C$ and $E$ ).

To evaluate overall trends with respect to the impact of $\mathrm{HI}$ on MBP immunostaining, MBP immunostaining in each cere-

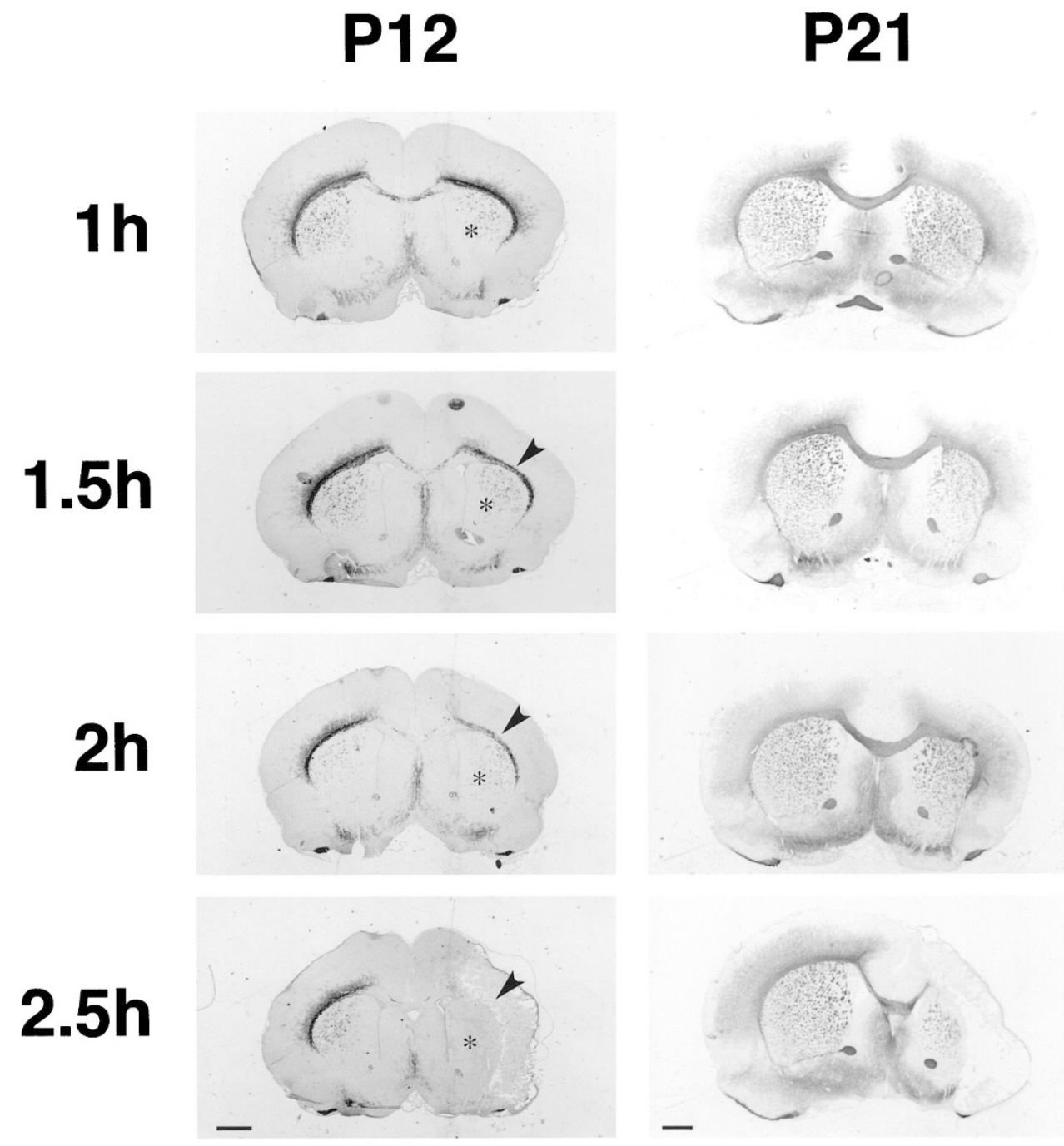

Figure 2. HI-induced disruption of MBP immunostaining. This photomontage compares the distribution of MBP immunostaining, at the level of the striatum, in coronal brain sections from eight animals, four evaluated on P12 (left), and four evaluated on P21 (right). All underwent right carotid artery ligation, followed by $8 \% \mathrm{O}_{2}$ exposure on $\mathrm{P} 7$; the duration of $8 \% \mathrm{O}_{2}$ exposure $(1,1.5,2$, or $2.5 \mathrm{~h})$ is indicated for each row. Only $2.5 \mathrm{~h}$ of $8 \% \mathrm{O}_{2}$ exposure resulted in ipsilateral infarction. In the P12 animals, loss of ipsilateral MBP immunostaining both in the external capsule (arrowhead) and adjacent striatum (*) becomes more pronounced with increasing duration of HI. In contrast, in the P21 animals MBP immunostaining is markedly attenuated only in the most severely lesioned brain. Scale bars, $1 \mathrm{~mm}$. 
P12

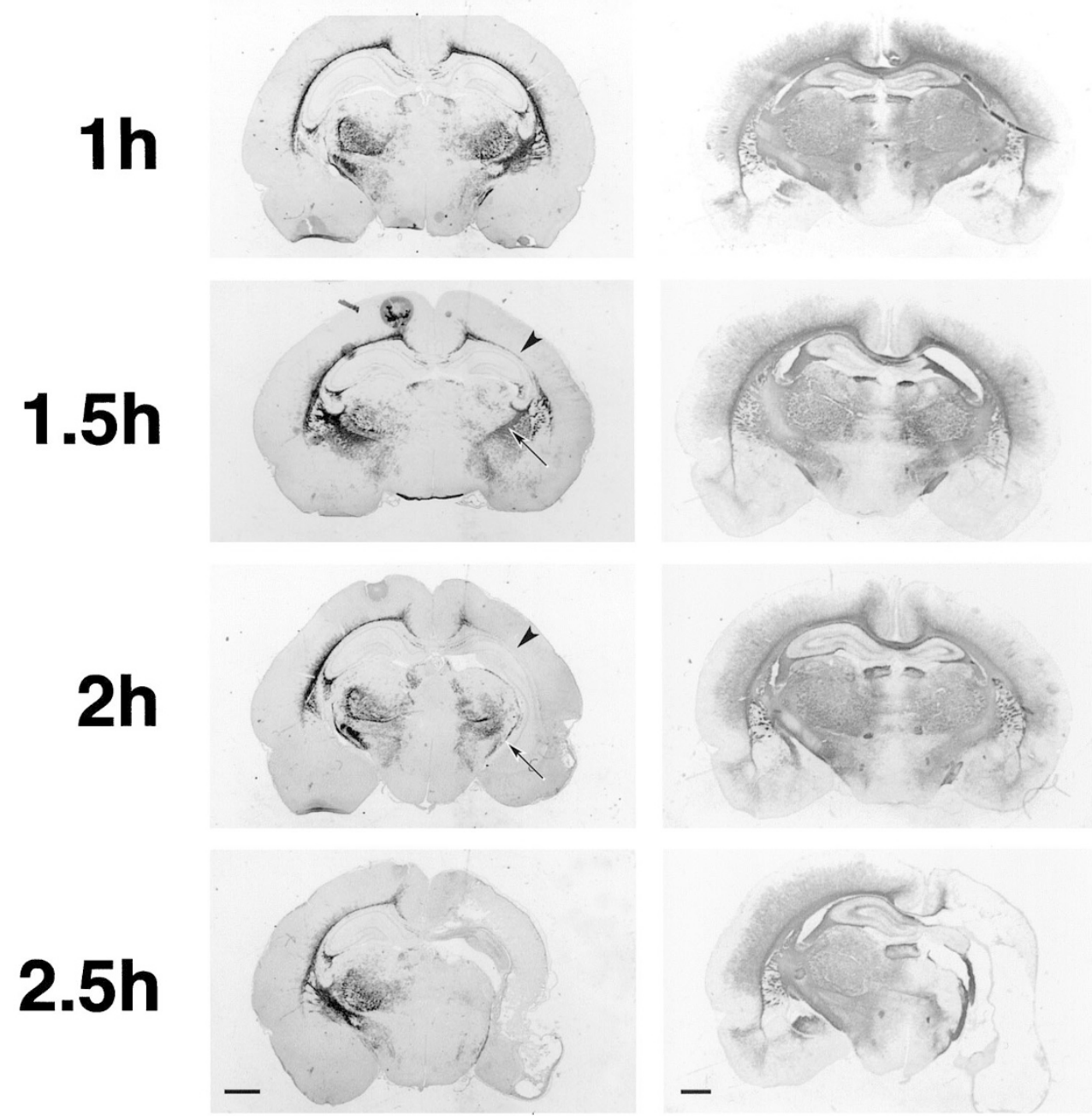

Figure 3. HI-induced changes in MBP immunostaining. This photomontage compares the distribution of MBP immunostaining, at the level of the mid-dorsal hippocampus, in coronal brain sections from eight animals, four evaluated on P12 (left), and four evaluated on P21 (right). Sections are from the same animals as in Figure 2. All underwent right carotid artery ligation, followed by $8 \% \mathrm{O}_{2}$ exposure on $\mathrm{P} 7$; the duration of $8 \% \mathrm{O}_{2}$ exposure $(1,1.5$, 2, or 2.5 h) is indicated for each row. Overall trends are congruent with the findings illustrated in Figure 2. In the P12 samples, there is a progressive loss of ipsilateral MBP immunostaining with increasing duration of HI, most notably in the external capsule (arrowhead) and internal capsule (arrow); in contrast, on P21, marked attenuation of MBP immunostaining is only discerned in the most severely lesioned animal. Scale bars, $1 \mathrm{~mm}$.

bral hemisphere was estimated by computerized densitometry

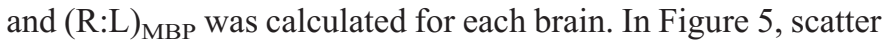
plots illustrate the distributions of these values in the P12 (Fig. $5, A$ ) and P21 (Fig. 5, B) outcome groups; median values are indicated by the horizontal bars. On P12, the median values in all four lesion groups were $<1.0$, and values were lowest in the 2.5-h HI animals; there were significant differences among groups ( $p<0.005$, Kruskal-Wallis test). On P21, in contrast, median values were $>1.0$ in both the 1 -h and 1.5 -h HI groups. Ratio values declined in the more severely lesioned animals, and there were significant intergroup differences $(p<0.025$, Kruskal-Wallis test).

Both the microscopic evaluation and the quantitative analysis suggested that there were differences between the patterns of MBP immunostaining resulting after mild HI lesioning on $\mathrm{P} 12$ versus $\mathrm{P} 21$. To evaluate this trend, we compared the extent of tissue atrophy and MBP immunostaining in the two groups of animals that had undergone $1.5 \mathrm{~h} \mathrm{HI}$. To confirm that the overall severity of injury was equivalent, area measurements were compared (Fig. 6A); there were no differences between the two groups $\left[(\mathrm{R}: \mathrm{L})_{\mathrm{AREA}}, \mathrm{P} 12,0.84 \pm 0.3 ; \mathrm{P} 21,0.90 \pm 0.0 ; p=\mathrm{NS}\right.$, Mann-Whitney test]. In contrast, there was a substantial difference in $(\mathrm{R}: \mathrm{L})_{\mathrm{MBP}}$ values between the two age groups (Fig. $6 B ; p<0.005$, Kruskal-Wallis test); on P12 the

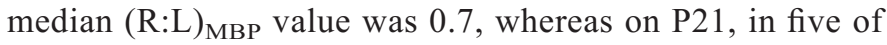
six animals the (R:L) $)_{\mathrm{MBP}}$ was $>1$ (i.e. more MBP immunostaining in the lesioned right hemisphere than contralaterally). In contrast, a similar analysis of more severely lesioned animals $(2.5 \mathrm{~h} \mathrm{HI})$ revealed no difference in the 

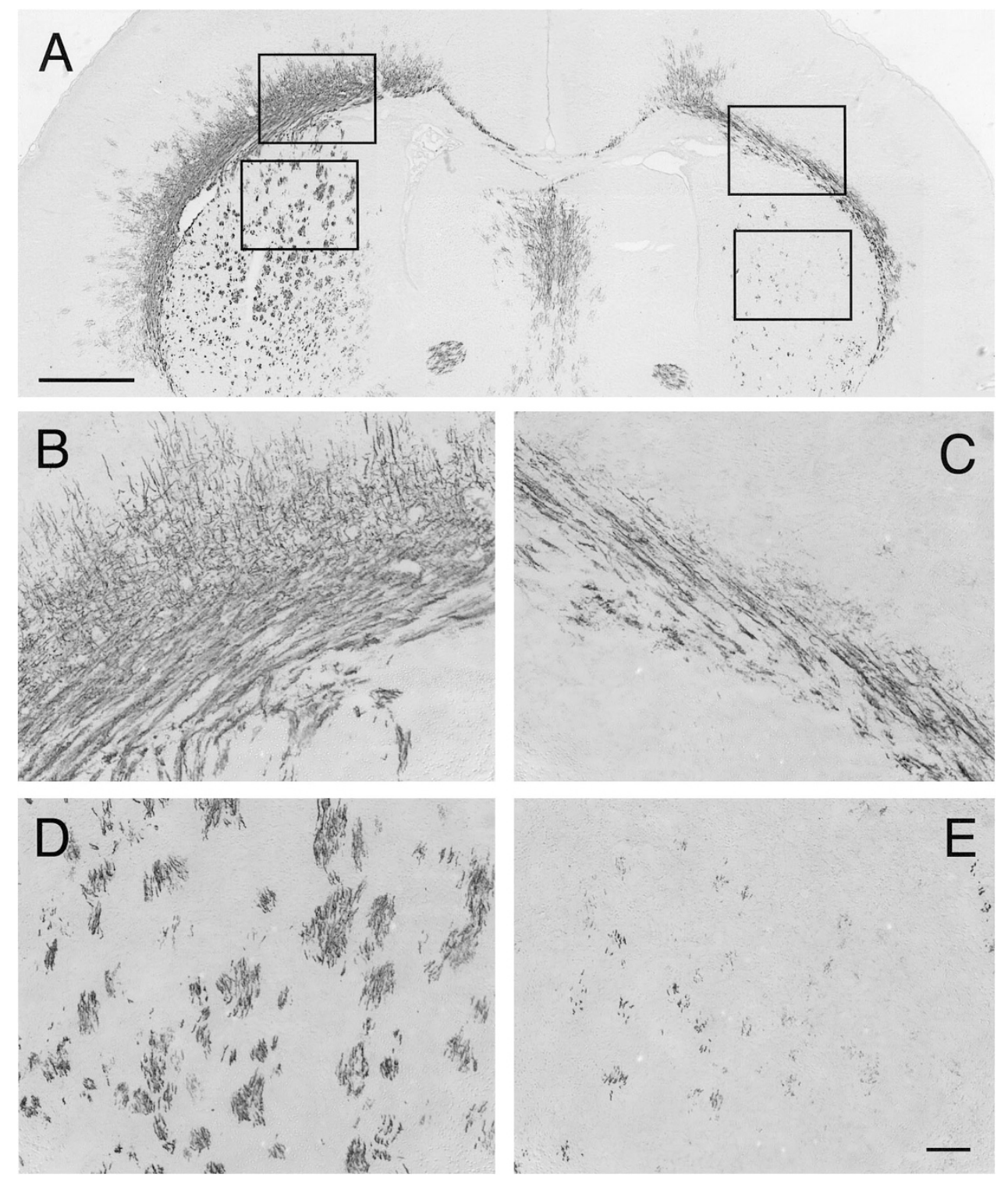

Figure 4. Early loss of MBP immunostaining in mildly lesioned animal. These images illustrate the disruption of MBP immunostaining in a representative P12 animal that underwent right carotid artery ligation, followed by $1.5 \mathrm{~h}$ of $8 \% \mathrm{O}_{2}$ exposure on P7. A, note that overall tissue integrity is preserved, although the lesioned right striatum is mildly atrophic; MBP immunostaining is reduced in the right external capsule, adjacent cortex, and striatum. $B-E$, higher magnifications of the four areas indicated in $A . B$ and $C$ compare corresponding areas of the left and right external capsule, and demonstrate the marked loss of MBP-immunostained axonal processes. $D$ and $E$ compare corresponding areas within the left and right striatum and illustrate, in transverse section, the marked reduction of MBP-immunoreactive axonal processes within the right striatum. Scale bars, $A, 1 \mathrm{~mm} ; B-E, 0.1 \mathrm{~mm}$.

degree of suppression of MBP between the P12 and P21 groups (data not shown).

\section{DISCUSSION}

Our data indicate that loss of MBP immunostaining provides a sensitive indicator of acute HI oligodendroglial injury in neonatal rodent brain. $\mathrm{HI}$ insults of increasing duration result in progressively widespread disruption of MBP immunostaining within $5 \mathrm{~d}$ after lesioning. However, during a 2-wk recovery period, some restoration of MBP immunostaining occurs in the absence of tissue infarction.

Our findings must be interpreted cautiously. The initial loss of MBP immunostaining reflects oligodendroglial dysfunction, but not necessarily cell death. Mechanisms that could contrib- ute to HI-induced loss of MBP immunostaining include reduced synthesis and increased degradation of MBP mRNA and protein.

In a closely related neonatal mouse model of $\mathrm{HI}$ injury, we found a very rapid suppression of oligodendroglial gene expression in the ipsilateral hemisphere (beginning as early as $3 \mathrm{~h}$ after insult, and progressively decreasing for up to $48 \mathrm{~h}$ ) (5). Our preliminary experiments to assay MBP mRNA in lesioned neonatal rat brain have yielded similar trends, i.e. $>50 \%$ decrease in MBP mRNA at 24-48 h after a severe HI insult (18). These results suggest that reductions in mRNAs encoding MBP and a subsequent decline in protein synthesis represent a major mechanism that contributes to loss of MBP immunostaining on P12. Activation of specific proteases by $\mathrm{HI}$ injury 
A.

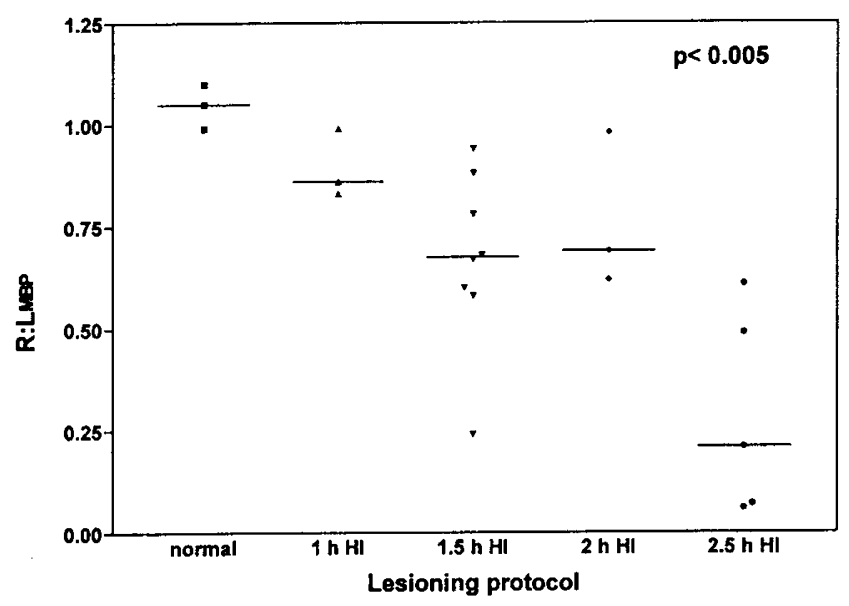

B.

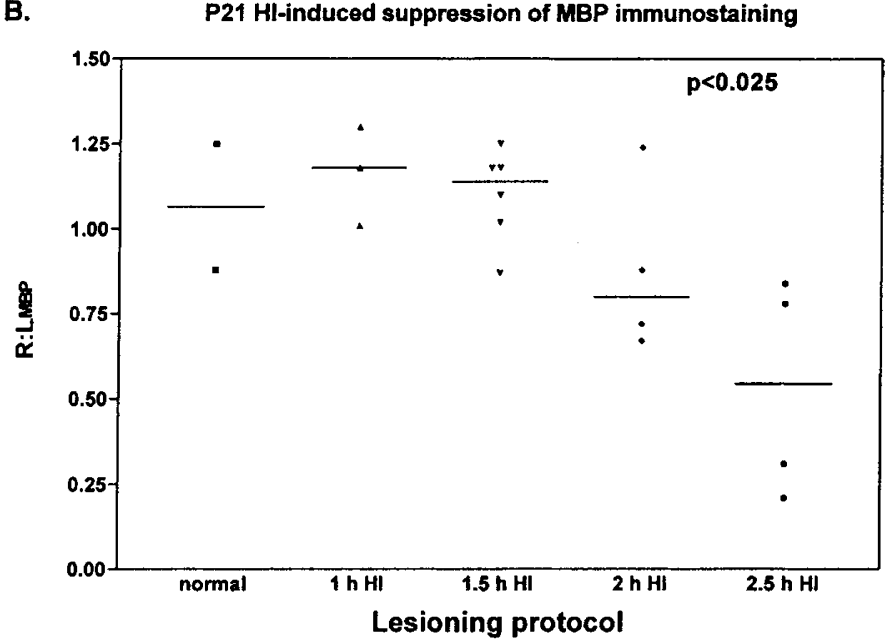

Figure 5. Densitometric analysis of MBP immunostaining. MBP immunostaining in each cerebral hemisphere was estimated by computerized densitometry (see "Methods"); for each brain sample, (R:L) ${ }_{\mathrm{MBP}}$ of pixels per right hemisphere to pixels per left hemisphere was calculated. $A$, scatterplot of these values in all $\mathrm{P} 12$ animals analyzed, sorted by duration of HI. Group medians are indicated by the horizontal bars; there were significant differences among groups $(p<0.005$, Kruskal-Wallis test). $B$, scatterplot of the corresponding values in P21 animals. There were significant intergroup differences ( $p<0.025$, Kruskal-Wallis test).

may also contribute to myelin degradation. Calpain degrades all major myelin proteins, and there is increased calpain activity after neonatal cerebral HI in this model (19). Increased calpain activity has also been documented in brain tissue from patients with multiple sclerosis, and is implicated as a factor contributing to myelin degradation in multiple sclerosis (20).

Multiple mediators could contribute to neonatal HI oligodendroglial injury. In this neonatal stroke model, HI injury elicits a widespread and complex inflammatory response (1315, 21-23). Clinical studies have implicated proinflammatory cytokines as mediators of perinatal white matter injury (24, 25). Supportive experimental data are provided by results of a recent study in which the inflammatory stimulus lipopolysaccharide was administered to pregnant rat dams (8); this intervention resulted in qualitative reductions in MBP immunostaining on $\mathrm{P} 8$, preceded by evidence of stimulation of cytokine expression in the fetal rat brain.

Compelling experimental data indicate that oligodendroglia are also highly susceptible to glutamate-mediated injury (1), both in the neonatal and adult rodent brain $(7,26)$. In the neonatal rodent stroke model used in this study, we have shown with in vivo microdialysis that glutamate accumulates extracellularly during the $\mathrm{HI}$ insult (27); this excitatory amino acid could contribute to white matter injury. Treatment with NBQX, an AMPA-type glutamate antagonist, substantially limited HI-induced loss of MBP immunostaining in a closely related neonatal stroke model (7); this finding provides strong support for the hypothesis that endogenous excitatory amino acids are directly toxic to immature oligodendroglia.

In the neonatal mouse stroke model, we found ultrastructural evidence that maturing, myelinating oligodendrocytes were normal, whereas some immature oligodendrocyte progenitors were dying within $3 \mathrm{~h}$ after the HI insult (5). Taken together, the immunocytochemical and ultrastructural data suggest that both death of oligodendrocyte progenitors and reduction of MBP mRNA and protein synthesis in surviving oligodendrocytes contribute to the initial loss of MBP immunostaining.
The inclusion of two outcome intervals in this study enabled us to evaluate sequential changes in MBP and to compare trends in animals that had incurred a relatively broad range of $\mathrm{HI}$ insults. The first time point, at P12, was selected based on our previous experience indicating that, in this model, the extent of neuronal loss and infarction could be reliably evaluated $5 \mathrm{~d}$ after lesioning (16). Yet, as the timing of the insult in this model of developmental brain injury coincides with a period of marked maturational changes in myelin production, we included a second time point to evaluate more long-term effects on myelin integrity. This approach enabled us to discern unexpected evidence of restoration of MBP immunoreactivity. Of interest, a recent study evaluating changes in expression of the oligodendroglial marker 2'-3'-cyclic nucleotide 3'phosphodiesterase, by Western blot assays of tissue homogenates, found that at $8-15 \mathrm{~d}$ after lesioning there were higher concentrations in the $\mathrm{HI}$ than in the contralateral hemisphere (28). These trends could reflect recovery of function or increased MBP synthesis by surviving oligodendroglia or proliferation and maturation of oligodendroglial precursors; future studies incorporating proliferation markers will be essential to evaluate the contributions of these mechanisms.

Both classic and modern studies indicate that new oligodendrocytes can be generated from oligodendrocyte precursors or from mature oligodendrocytes in the adult CNS $(29,30)$. For example, Ludwin (30) reported, in the late 1970s, evidence of proliferation of mature oligodendrocytes, after cuprizoneinduced demyelination in the adult CNS. More recent studies have confirmed that adult animals harbor an abundant population of oligodendrocyte precursor cells that slowly generate new oligodendrocytes (31) and respond to experimentally induced brain injury (32). In a study using a model of middle cerebral artery occlusion to induce focal ischemic injury in adult mouse brain, Mandai et al. (33) found a reduction in number of oligodendrocytes expressing myelin mRNAs shortly after injury but an increase in number of oligodendrocytes at later time points. These findings may indicate subse- 
A. Comparison of atrophy in mildly lesioned groups

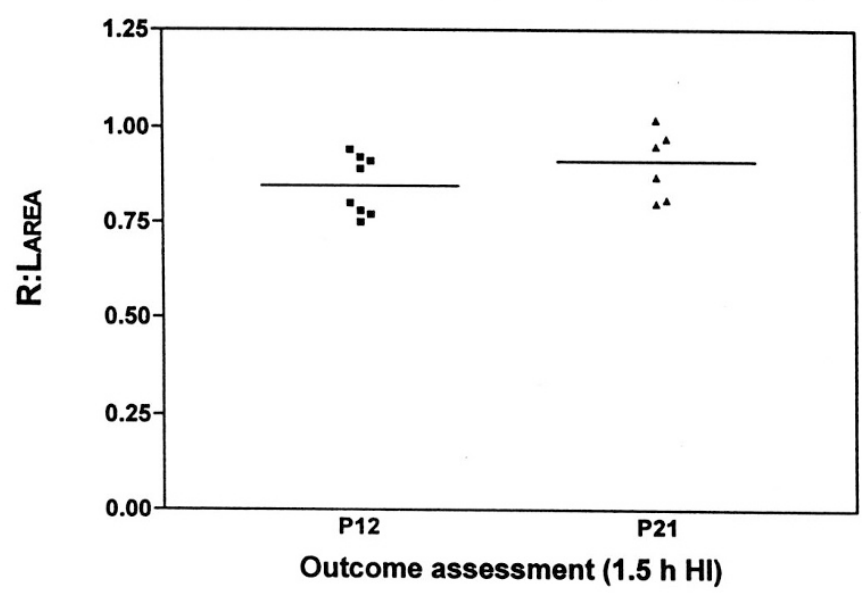

B. MBP immunostaining in mildly lesioned groups

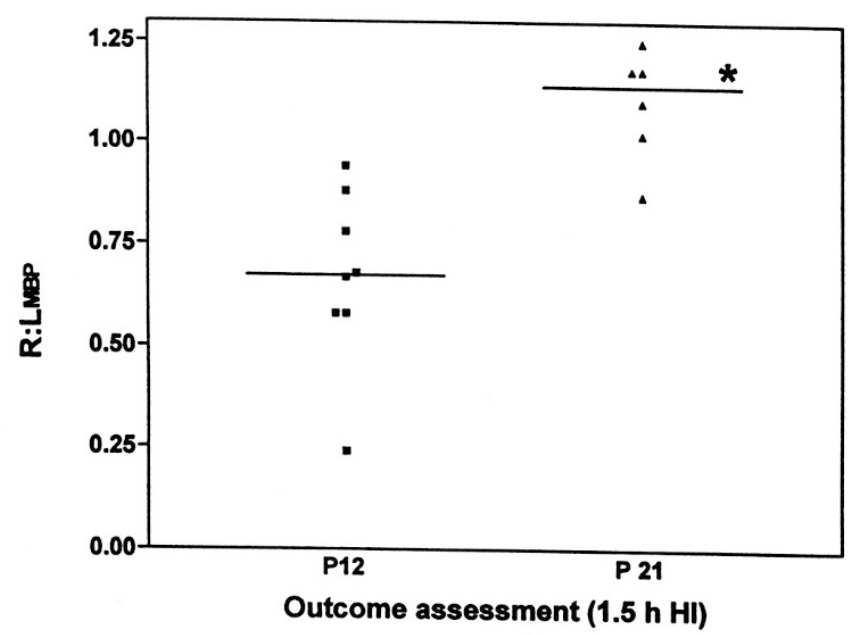

Figure 6. Comparison of extent of tissue injury and loss of MBP immunostaining in mildly lesioned animals. These graphs compare the extent of tissue injury, expressed as $(\mathrm{R}: \mathrm{L})_{\mathrm{AREA}}(A)$ and $(\mathrm{R}: \mathrm{L})_{\mathrm{MBP}}(B)$ in mildly lesioned animals (right carotid artery ligation, followed by $1.5 \mathrm{~h}$ of $8 \% \mathrm{O}_{2}$ exposure on P7) on P12 and P21. There were no differences in the severity of injury between the two groups ( $p=\mathrm{NS}$, Mann-Whitney test). In contrast, there was a substantial difference in (R:L) $)_{\text {MBP }}$ values between the two age groups $(p<$ 0.005 , Kruskal-Wallis test); in particular, note that on P21, in five of six animals the (R:L) $)_{M B P}$ value was $>1$.

quent proliferation of oligodendrocytes, as suggested by the authors. However, their findings could also reflect upregulation of myelin gene expression in surviving oligodendrocytes, as proliferation studies were not performed.

Even though MBP immunostaining is relatively restored at P21 in mildly lesioned animals, our data provide no information about regeneration of intact myelin sheaths. Loss of myelin, even transiently, can markedly disrupt CNS development by interfering with axonal function, and perhaps neuronal survival. A recent clinical study provided compelling evidence of the deleterious effect of periventricular white matter injury in the human neonate on subsequent cerebral cortical development (6). A quantitative volumetric magnetic resonance imaging technique was used to measure brain tissue volumes, at term, in premature infants with earlier diagnosis of periventricular leukomalacia; these infants had a marked reduction in cerebral cortical gray matter at term, compared with either premature infants without periventricular leukomalacia or normal term. This finding provides a strong rationale for the concurrent assessment of neuronal and oligodendroglial integrity in experimental models of perinatal brain injury.

These data demonstrate that oligodendroglia are highly susceptible to $\mathrm{HI}$ injury in the immature rodent brain. Quantitative MBP immunoassays provide a sensitive indicator of oligodendroglial injury. Our unexpected finding that relatively mild and more severe HI insults elicit divergent responses illustrates some of the complexities inherent in experimental analysis of oligodendroglial injury and repair in vivo. These results strongly suggest that after mild $\mathrm{HI}$ insults myelin markers are up-regulated in the lesion territory; in more severely lesioned tissue, there may be a similar potential for recovery in regions adjacent to the infarct territory. Whether specific therapeutic interventions can enhance recovery of oligodendroglial function is an important question for future research.

\section{REFERENCES}

1. Oka A, Belliveau MJ, Rosenberg PA, Volpe JJ 1993 Vulnerability of oligodendroglia to glutamate: pharmacology, mechanisms, and prevention. J Neurosci 13:1441-1453

2. Back SA, Gan X, Li Y, Rosenberg PA, Volpe JJ 1998 Maturation-dependent vulnerability of oligodendrocytes to oxidative stress-induced death caused by glutathione depletion. J Neurosci 18:6241-6253

3. Volpe JJ 1998 Brain injury in the premature infant: overview of clinical aspects, neuropathology, and pathogenesis. Semin Pediatr Neurol 5:135-151

4. Fern R, Moller T 2000 Rapid ischemic cell death in immature oligodendrocytes: a fatal glutamate release feedback loop. J Neurosci 20:34-42

5. Skoff RP, Bessert DA, Barks JDE, Song D, Cerghet M, Silverstein FS 2001 Hypoxic-ischemic injury results in acute disruption of myelin gene expression and death of oligodendroglial precursors in neonatal mice. Int J Dev Neurosci 19:197-208

6. Inder TE, Huppi PS, Wakefield S, Kikinis R, Zientara GP, Barnes PD, Jolesz FA, Volpe JJ 1999 Periventricular white matter injury in the premature infant is associated with a reduction in cerebral cortical gray matter volume at term. Ann Neurol 46:755-760

7. Follett PL, Rosenberg PA, Volpe JJ, Jensen FE 2000 NBQX attenuates excitotoxic injury in developing white matter. J Neurosci 20:9235-9241

8. Cai Z, Pan ZL, Pang Y, Evans OB, Rhodes PG 2000 Cytokine induction in fetal rat brains and brain injury in neonatal rats after maternal lipopolysaccharide administration. Pediatr Res 47:64-72

9. Dommergues MA, Patkai J, Renauld JC, Evrard P, Gressens P 2000 Proinflammatory cytokines and interleukin- 9 exacerbate excitotoxic lesions of the newborn murine neopallium. Ann Neurol 47:54-63

10. Han BH, D'Costa A, Back SA, Parsadanian M, Patel S, Shah AR, Gidday JM, Srinivasan A, Deshmukh M, Holtzman DM 2000 BDNF blocks caspase-3 activation in neonatal hypoxia-ischemia. Neurobiol Dis 7:38-53

11. Rice JE, Vannucci RC, Brierley JB 1981 The influence of immaturity on hypoxicischemic brain damage in the rat. Ann Neurol 9:131-141

12. Vannucci RC, Rossini A, Towfighi J, Vannucci S 1997 Measuring the accentuation of the brain damage that arises from perinatal cerebral hypoxia-ischemia. Biol Neonate 72:187-191

13. Towfighi J, Zec N, Yager J, Housman C, Vannucci RC 1995 Temporal evolution of neuropathologic changes in an immature rat model of cerebral hypoxia: a light microscopic study. Acta Neuropath 90:375-386

14. Ivacko JA, Sun R, Silverstein FS 1996 Hypoxic-ischemic brain injury induces an acute microglial reaction in perinatal rats. Pediatr Res 39:39-47

15. Burtrum D, Silverstein FS 1994 Hypoxic-ischemic brain injury stimulates glial fibrillary acidic protein mRNA and protein expression in neonatal rats. Exp Neurol 126:112-118

16. Liu XH, Eun BL, Silverstein FS, Barks JDE 1996 The platelet-activating factor antagonist BN 52021 attenuates hypoxic-ischemic brain injury in the immature rat. Pediatr Res 40:797-803

17. Mujsce DJ, Christensen MA, Vannucci RC 1990 Cerebral blood flow and edema in perinatal hypoxic-ischemic brain damage. Pediatr Res 27:450-453

18. Xu H, Liu YQ, Skoff R, Barks JD, Silverstein FS 2000 Acute oligodendroglial injury in neonatal rodent brain. Pediatr Res 47:467A(abstr)

19. Blomgren K, Hallin U, Andersson AL, Puka-Sundvall M, Bahr BA, McRae A, Saido TC, Kawashima S, Hagberg H 1999 Calpastatin is up-regulated in response to hypoxia and is a suicide substrate to calpain after neonatal cerebral hypoxia-ischemia. J Biol Chem 274:14046-14052 
20. Shields DC, Schaecher KE, Saido TC, Banik NL 1999 A putative mechanism of demyelination in multiple sclerosis by a proteolytic enzyme, calpain. Proc Natl Acad Sci USA 96:11486-11491

21. Szaflarski J, Burtrum D, Silverstein FS 1995 Cerebral hypoxia-ischemia stimulates cytokine gene expression in perinatal rats. Stroke 26:1093-1100

22. Ivacko J, Szaflarski J, Malinak C, Flory C, Warren JS, Silverstein FS 1997 Hypoxicischemic injury induces monocyte chemoattractant protein-1 expression in neonatal rat brain. J Cereb Blood Flow Metab 17:759-770

23. Bona E, Andersson AL, Blomgren K, Gilland E, Puka-Sundvall M, Gustafson K, Hagberg H 1999 Chemokine and inflammatory cell response to hypoxia-ischemia in immature rats. Pediatr Res 45:500-509

24. Perlman JM, Risser R, Broyles RS 1996 Bilateral cystic periventricular leukomalacia in the premature infant: associated risk factors. Pediatrics 97:822-827

25. Dammann O, Leviton A 1997 Maternal intrauterine infection, cytokines, and brain damage in the preterm newborn. Pediatr Res 42:1-8

26. McDonald JW, Althomsons SP, Hyrc KL, Choi DW, Goldberg MP 1998 Oligodendrocytes from fore-brain are highly vulnerable to AMPA/kainate receptor-mediated excitotoxicity. Nat Med 4:291-297
27. Gordon K, Simpson J, Statman D, Silverstein FS 1991 Effects of perinatal stroke on striatal amino acid efflux in rat studied with in vivo microdialysis. Stroke 22:928-932

28. Cheepsunthorn P, Palmer C, Menzies S, Roberts RL, Connor JR 2001 Hypoxic/ ischemic insult alters ferritin expression and myelination in neonatal rat brains. J Comp Neurol 431:382-396

29. Herndon R, Price DL, Weiner L 1977 Regeneration of oligodendroglia during recovery from demyelinating disease. Science 195:693-694

30. Ludwin SK 1978 Central nervous system demyelination and remyelination in the mouse: an ultrastructural study of cuprizone toxicity. Lab Invest 39:597-612

31. Levison SW, Young GM, Goldman JE 1999 Cycling cells in the adult rat neocortex preferentially generate oligodendroglia. J Neurosci Res 57:435-446

32. Keirstead HS, Blakemore WF 1999 The role of oligodendrocytes and oligodendrocyte progenitors in CNS remyelination. Adv Exp Med Biol 468:183-197

33. Mandai K, Matsumoto M, Kitagawa K, Matsushita K, Ohtsuki T, Mabuchi T, Colman DR 1997 Ischemic damage and subsequent proliferation of oligodendrocytes in focal cerebral ischemia. Neuroscience 77:849-886 